\title{
Do you believe that atoms stay in place when you observe them in HREM?
}

\author{
Dirk Van Dyck ${ }^{\mathrm{a}}$ Ivan Lobato ${ }^{\mathrm{b}}$, F.-R. Chen ${ }^{\mathrm{c}}$ and C. Kisielowski ${ }^{\mathrm{d}}$
}

${ }^{a}$ EMAT, University of Antwerp, Groenenborgerlaan 171, 2020 Antwerpen, Belgium, e-mail: dirk.vandyck@uantwerp.be

${ }^{\mathrm{b}}$ EMAT, University of Antwerp, Groenenborgerlaan 171, 2020 Antwerpen, Belgium, e-mail: ivan.lobato@uantwerp.be

${ }^{c}$ Dept. of Engineering and System Science, National Tsing Hua University, Number 101, Section 2, Kuang-Fu Road, Hsin Chu, Taiwan 300, China, e-mail: frchen1@me.com

${ }^{\mathrm{d}}$ National Center for Electron Microscopy and Joint Center for Artificial Photosynthesis, Lawrence Berkeley National Laboratory, One Cyclotron Rd., Berkeley, CA 94720, USA, e-mail: cfkisielowski@lbl.gov

Corresponding author:

Dirk Van Dyck

EMAT, University of Antwerp

Groenenborgerlaan 171

2020 Antwerpen, Belgium

e-mail: dirk.vandyck@uantwerp.be

Tel. +32 32653258 


\begin{abstract}
Recent advancements in aberration-corrected electron microscopy allow for an evaluation of unexpectedly large atom displacements beyond a resolution limit of $\sim 0.5 \AA$, which are found to be dose-rate dependent in high resolution images. In this paper we outline a consistent description of the electron scattering process, which explains these unexpected phenomena. Our approach links thermal diffuse scattering to electron beam-induced object excitation and relaxation processes, which strongly contribute to the image formation process. The effect can provide an explanation for the well-known contrast mismatch ("Stobbs factor") between image calculations and experiments.
\end{abstract}

Keywords: Quantitative HREM, TDS, Dose rate dependence

\title{
1. Introduction
}

The fact that atoms vibrate during observation in an electron microscope is generally known. But the effects of this atom motion on the formation of high-resolution images is less understood. It is even not clear to what extent this motion is driven by the temperature of the specimen or by the energy transfer from the inelastic scattering of the incident electrons.

In X-ray diffraction where the interaction between the photons and the atoms is weak, the X-ray beams see a kind of "spatially averaged" atom that is equivalent to a "time averaged" atom. This "blurring" of the atom is then accounted for by multiplying the scattering factor with a DebyeWaller factor. This is common practice in all X-ray programs for quantitative structure refinement. On the other hand, in electron microscopy, the electrons are so fast that they see "frozen" atoms displaced from their equilibrium position. The image is then a time average over the different atom positions which is not equivalent to an image formed with a time average of the atomic potential [1]. Moreover, the electrons interact also with the strong electrostatic potential of the atom nucleus and are scattered over much larger angles than X-rays. By simply multiplying the atomic scattering factor with a Debye-Waller factor one artificially "smoothens" the atom potential and erroneously suppresses the high angle thermal diffuse scattering (TDS) The only correct way to include the thermal motion of the atoms in the image simulations is by using a frozen phonon model in which the calculations are repeated for various configurations of still atoms, displaced from their equilibrium positions and by averaging the image intensities afterwards. This is common practice in nearly all programs for accurate quantitative HAADF STEM simulations [2] where the main part of the signal is generated by thermal diffuse scattering.

\section{Thermal diffuse scattering in HRTEM}

Hÿtch and Stobbs [3] were the first to point out that even the most realistic computer image simulations for simple known crystalline objects do not match with the best experimental micrographs in the sense that the overall contrast is wrong by a factor of about three. This means that an important aspect of electron scattering or imaging is still not properly accounted for in the image simulation programs. In view of its importance, this "Stobbs factor" problem has been 
studied extensively over more than one decade [4-14] with many clever and careful experiments to eliminate possible causes but despite these efforts no decisive explanation has yet been given. Nevertheless, thermal diffuse scattering is still left over as a possible cause for the following reasons:

- The Stobbs factor remains present in sub-eV energy filtered HREM images [4] which rules out inelastic plasmon and core loss scattering. This leaves only inelastic interactions in the sub-eV range such as phonon scattering.

- Quantitative electron diffraction methods such as CBED [15], MSLS [16] and precession [17] do not suffer from mismatch with simulations. The reason is that they subtract the thermal diffuse background from the Bragg reflections.

- With increasing crystal thickness, not only the contrast of HREM images decreases but also the intensity. The intensity loss can be around 20\% [18], which means that up to $20 \%$ of the electrons do not reach the image. This can be explained by phonon scattering since a large fraction of the inelastic electrons are scattered outside the physical apertures of the electron microscope and are eliminated from the image.

- Experimental measurements also estimate that the total diffuse intensity in diffraction patterns is of the order of $10 \%$ to $20 \%$ [19], which seems small compared to the intensities in Bragg spots. However, the diffuse intensity is distributed all across reciprocal space so that its total contribution can still be significant.

- The contrast reduction in an image is not only due to an increase of background intensity but dominantly caused by a reduction of the elastic signal itself so that even small contributions from phonon scattering can cause strong contrast alterations in the image since the underlying relations are non-linear.

\section{Au [110] - Vacuum wave}
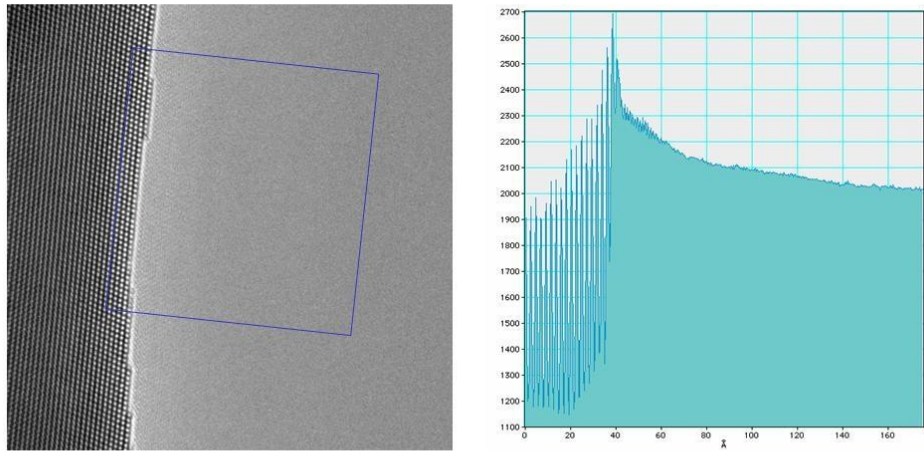

Courtesy C. Kisielowski, J.R. Jinschek (NCEM, Berkeley)

Figure 1. HREM image of a Au [110] showing a tail of diffuse intensity that protrudes a long distance in the vacuum [7]. Far from the sample inside the vacuum the intensity is that of the incident beam. But inside the sample the intensity is lower, thus part of the intensity is missing and it reappears in the vacuum region close to the sample where the intensity is higher than that of the incident beam. Thus the intensity is "leaking" out of the sample into the vacuum. 
-Figure 1 shows that in the case of $\mathrm{Au}$ [110] a significant fraction of the imaging electrons is delocalised over a long distance beyond the edge of the sample. In principle one can envisage different causes such as the presence of coma, but we believe that it is also consistent with the TDS picture.

\section{HRTEM Image simulations}

Contrary to HAADF simulation programs none of the standard HRTEM simulation programs apply the frozen phonon model. Moreover they often include the incoherent aberrations of the electron microscope as a damping envelope on the transfer function instead of calculating the images corresponding with the fluctuating parameters of the electron microscope separately and averaging afterwards. With this damping envelope approximation the phase oscillations at the large spatial frequencies are artificially suppressed so that the TDS electrons are not delocalised in the background but keep contributing to the contrast. The reason why HRTEM simulation programs do not use a frozen phonon model nor a full integration over the incoherent EM aberrations is mainly because they are very time consuming and their importance for HRTEM is not fully appreciated. But as argued in [1] we believe that this can be a reason for the "Stobbs factor".

In a recent paper [5] it was suggested that the Stobbs factor is only due to an underestimation of the Modulation Transfer Function (MTF) of the camera. And as already reported in 2001 by O'Keefe et al. (Ultramicroscopy) this can indeed cause a reduction in contrast. But there are several reasons why one can doubt that the MTF would be the only cause of reduction in contrast. Since the MTF of a camera is a convolution operation on the image intensity that only redistributes the intensity it cannot explain the loss of intensity as observed in Figure 1. And in one of the papers in [4] the MTF of the camera has been measured meticulously and included in the simulations but it could not explain the Stobbs factor.

Our main arguments against the conclusions in [5] are that the experimental HRTEM images of thin $\mathrm{SrTiO}_{3}$ are compared with computer simulations using Debye-Waller factors instead of a frozen phonon model so that the importance of the thermal atom motion on the image contrast is not properly included.

We therefore have repeated these simulations for exactly the same object $\left(\mathrm{SrTiO}_{3}\right)$ and experimental conditions, but now using a proper frozen phonon model and a correct treatment of the incoherent aberrations. The results are shown in Figure 2. And although these simulations are indeed very time consuming they are worth the effort. Indeed the results show that by using a frozen phonon model one can indeed also reduce the contrast by a factor of 2 to 4 which, in addition to the MTF considerations could explain at least a part of the Stobbs factor. However a Stobbs factor of 3 would then correspond with an average atom displacement of about $0.5 \AA$ which is much larger than the estimated atom displacement of $0.1 \AA$ to $0.2 \AA \mathrm{pm}$ at room temperature [14]. We believe that the reason has to be sought in the energy that is transferred and accumulated to the atoms by the inelastic collision of successive incident electrons. If this hypothesis would be true, one can expect that the average displacement of the atoms would decrease with decreasing dose rate of the illumination and that the elastic contrast would consequently increase. In the following we wilt test this hypothesis. We do not disagree that one needs to include the MTF in image simulations. But we explicitly want to make the point that the 
contrast can be altered by a factor of 2 simply by changing the incident electron dose rate, only, with all other microscope parameters kept constant. This is why our detailed discussions furtheron focus on the physics of the atom motions and relaxation processes which may or may not modify the image formation process and not on cameras,

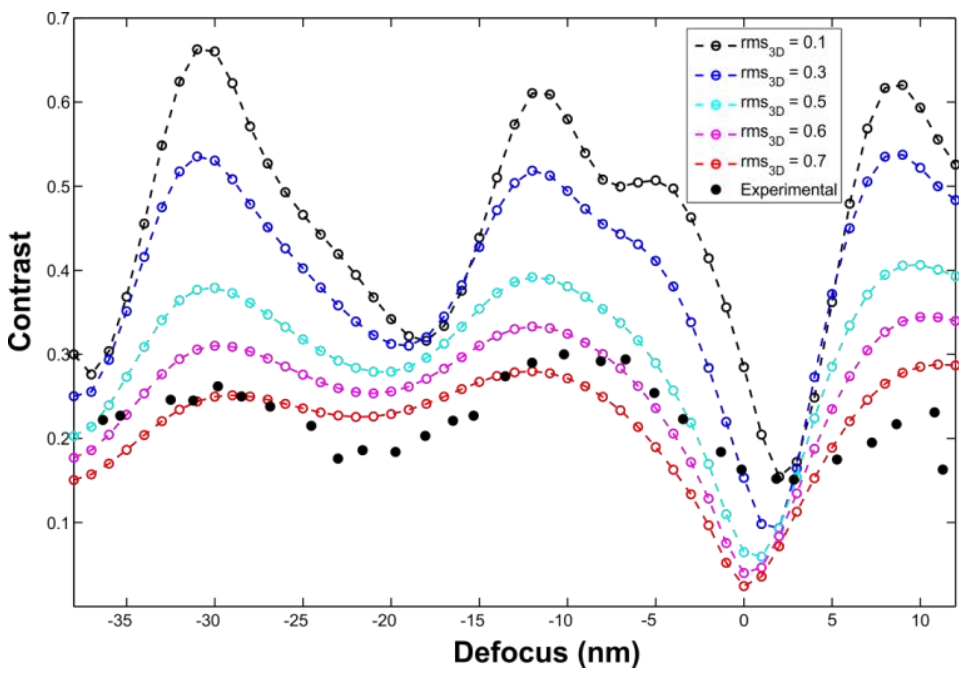

Figure 2. HRTEM Image contrast simulated for a $2.8 \mathrm{~nm}$ thick of $\mathrm{SrTiO}_{3}[110]$ against defocus with the following electron microscope setting: acceleration voltage $\left(E_{0}=300 \mathrm{KeV}\right)$, defocus spread $(\Delta=3.3 \mathrm{~nm})$, semi-convergence angle $(\alpha=0.25 \mathrm{mrad})$, spherical aberration $\left(C_{s}=-0.025 \mathrm{~mm}\right)$. A super cell size was used of dimensions $23.43 \AA \times 22.09 \AA \times 27.61 \AA$ which contain 1497 atoms, sampled in a numerical real space grid of $1536 \times 1536$ pixels. This corresponds to a real space sampling resolution equal to $0.0864 \AA$ and a sampling in reciprocal space equal to $0.045 \AA^{-1}$. To avoid aliasing, the electron wave function and the transmission function at each slice of the calculation are bandlimited to $g_{\max }=21.85 \AA^{-1}$. The frozen phonon simulation was performed by using the Einstein model with 60 configurations, slice thickness of 0.35 A and with different root mean square (rms) of the atom displacement in its 3D vibration. In case of a gaussian position distribution the rms is proportional to the square root of the Debye-Waller factor. The spatial and temporal incoherence are included by repeating the multislice calculation of each frozen phonon configuration for each angle in the condenser aperture and integrating the intensity over a range of defocus values of the wave function convolved with the objective lens point spread function.

\section{Is thermal diffuse scattering elastic or inelastic?}

In HAADF simulations one uses a multislice code in which the atoms are frozen at their displaced positions. But a multislice simulation describes only the elastic interaction between the electron and the crystal. The diffuse scattering is generated by the fact that the atoms are quasi randomly displaced from their crystallographic equilibrium positions. And these atom positions are determined by phonon calculations at the given temperature of the object. Consequently, if one would lower the temperature to absolute zero the atoms would be fixed at their equilibrium position (apart from the zero point motion) and then the simulated TDS signal would disappear. On the other hand Rutherford's theory shows that beyond a certain diffraction angle the electrons unavoidably transfer energy to the atoms and make them vibrate. And this diffuse inelastic 
scattering does not interfere with the elastic wave. Thus a fraction of the TDS scattering is elastic and a fraction is inelastic.

The correct way to describe thermal diffuse scattering properly is in the framework of a true theory for inelastic scattering which incorporates both the electron and the quantum states of the crystal $[21,22]$.

In $[6,7]$ it is proven that the frozen phonon model for image simulations is in principle equivalent to a full quantummechanical calculation. But in this proof one implicitly assumes that the energy transfer to the crystal is small enough to leave the crystal unaltered.

But even if the probability for inelastic scattering is small as compared with the elastic scattering it has to be multiplied with the total number of illuminating electrons, which for HRTEM can be very large. Hence the total transferred energy accumulates and might cause the very large observed average displacement.

\section{Energy transfer by the imaging electrons}

The average energy transfer for a collision between an incident electron with energy $\mathrm{E}$ and the nucleus of an atom with atomic number $Z$ can be calculated as follows: According to Rutherford's theory the energy $\varepsilon$ transferred in the collision with scattering angle $\theta$ is given by

$$
\varepsilon=T \sin ^{2}(\theta / 2)
$$

with $T$ the maximal energy transfer given by

$$
T=E / 1000 Z
$$

where we have approximated (atom mass)/(electron mass) $=2000 \mathrm{Z}$

The total cross section for inelastic scattering with energy transfer between the minimal energy $\varepsilon$ and the maximal energy $T$ (with $T \gg \varepsilon$ ) is a circular disk with radius

$$
R=(\alpha Z / E) \sqrt{ }(T / \varepsilon)
$$

with

$$
\alpha=0.73 \mathrm{eV} / \mathrm{nm}
$$

and the average energy transfer is then

$$
\langle\varepsilon>=\varepsilon \ln (T / \varepsilon)
$$

The minimal energy transfer to excite a phonon is of the order of

$$
\varepsilon=1 / 4 k \theta_{D}
$$

with $k$ the Boltzmann factor and $\theta_{D}$ the Debye temperature of the material.

For a typical case with $E=300 \mathrm{keV}, Z=14$ and $\theta_{D}=200 \mathrm{~K}$, the maximal and minimal energy transfers are then respectively $T=20 \mathrm{eV}$ and $\varepsilon=4.5 \mathrm{meV}$. 
From (1) the minimal energy transfer corresponds with a scattering angle of $\theta=30 \mathrm{mrad}$ which is larger than the typical angles for elastic scattering. The radius of the cross section is then $R=$ $0.025 \mathrm{~nm}$ which is much smaller than the cross section for elastic scattering and the average energy transfer in the collision is $\langle\varepsilon\rangle=40 \mathrm{meV}$.

Since it takes a large number of electrons to image individual atoms at high resolution, and every electron transfers on average an energy $\langle\varepsilon\rangle$ to the atom, the transferred power density (energy per unit time and area) is proportional to the dose rate. It can be assumed that a significant fraction of the energy is dissipated by radiation or by heat conduction, which in a stationary situation is proportional to the thermal gradient. But this subtle balance is critically dependent both on the geometry and thermal contacts of the sample and on the dose rate of the illumination. Usually the samples used in HREM are very thin and the thermal contacts are poor so that the heat conduction can be very low and the local temperature in the sample can become relatively high. This can increase the thermal vibration of the atoms with a strong effect on the elastic thermal diffuse scattering (TDS) and on the contrast of HREM images.

The effect of electron beam induced sample heating is extensively debated in literature ever since it became known that nano particles are strongly altered during observation at high resolution [23]. For example, in [24] it was calculated that temperature variations over orders of magnitude are expectable depending on beam current density and thermal conductivity of the observed material. Thus, it is expectable that pristine objects structures are observed if beam currents are low and the related energy is effectively removed by thermal conduction. However, atomistic details of such relaxation processes are being debated now.

\section{Molecular dynamics simulations}

In order to estimate the influence of the increasing temperature of the specimen on the thermal vibrations of the atoms we could use a simple harmonic oscillation model. However this model breaks down at high temperatures where the vibrations become highly anharmonic or even start to break bonds.

In order to get a more accurate and quantitative understanding, we have performed molecular dynamics calculations for a single graphene layer at various temperatures between.

Figure 3a shows the perfect crystallographic structure of a single graphene layer. Figure 3 shows a single graphene layer with random $30 \mathrm{pm}$ large displacements of the atoms from their equilibrium positions. Figure $3 \mathrm{c}$ shows the histogram of the calculated atom displacements in a single layer graphene using Monte Carlo simulations at different temperatures between 0.20 and 0.75 of the melting temperature . The data are taken from Ref. [23].

At lower temperature, the histogram is symmetric with a maximum around 10 to $20 \mathrm{pm}$ which corresponds to the normal Debye-Waller factors. However, as the temperature of the simulation increases, the histogram becomes increasingly skewed. In the tail of the distribution, large displacements are present. They are related to the occurrence of single interstitial atoms [27] in two-dimensional layers [28] or from atoms in small crystals or on surfaces where the binding energies are lowered [26] or rare events such as a local formation of carbon chains (carbene) from graphene [25]. They can be observed directly in an HRTEM with a resolution of the order 
of $50 \mathrm{pm}$ (e.g. TEAM 0.5 Berkeley). As explained above, the high temperature can be the result of the accumulated energy from the inelastic scattering of successive electrons which is then released in thermalization processes. Such reversible or irreversible relaxation processes are indeed observed in recent time resolved experiments close to atomic resolution [32] and can directly be detected at ultra-high resolution in high performance microscopes $[31,33]$.
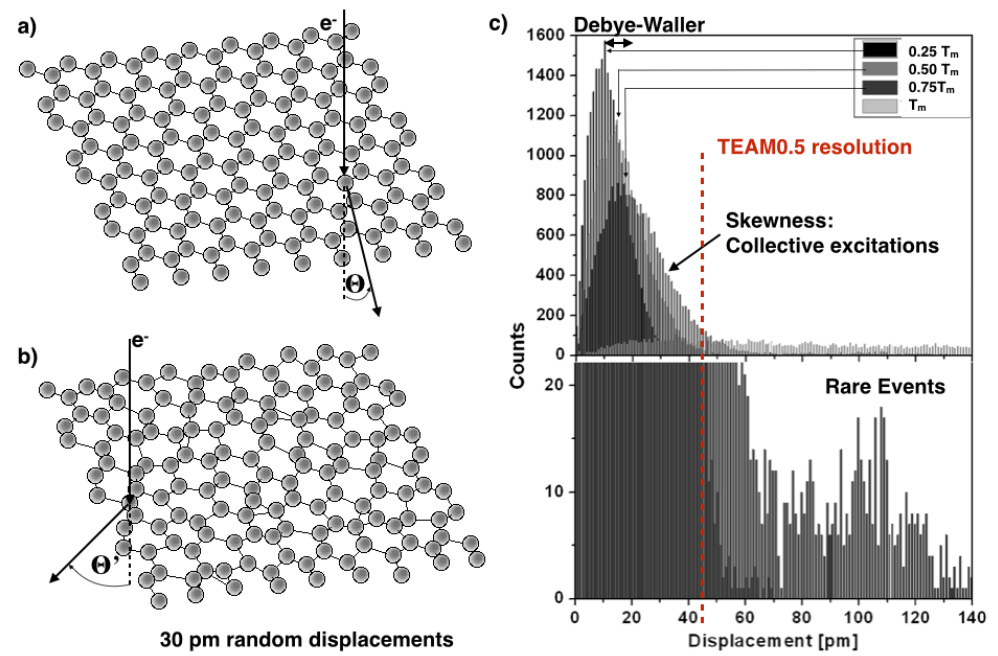

Figure 3. a) Single graphene layer with full crystallographic symmetry. b) single graphene layer with random $30 \mathrm{pm}$ large displacements of the atoms from their equilibrium positions. c) Calculated atom displacements in a single layer graphene using Monte Carlo simulations at different temperatures. $T_{m}$ is the melting point temperature and the dotted line marks the revolution limit of TEAM 0.5 electron microscope. Data are taken from Ref. [23].

Furthermore, increased thermal energies can promote the formation of defects or even materials disintegration no matter whether samples are considered radiation resistant or not. An important consequence of this consideration is that radiation damage is not an unavoidable consequence of the electron dose but can be reduced by using lower dose rates [26]. Moreover a controllable dose rate be used as an extra parameter to study the dynamics of physical processes such as phase transformations [29].

\section{Experimental evidence of dose rate dependence of image contrast.}

When an electron passes through an atom, the electron wave undergoes a phase shift that is proportional to the projected electrostatic potential of the atom. When the atom is "blurred" by the thermal vibrations, the projected potential is smoothened and the phase change is reduced especially close to the center of the atom. And since in our hypothesis, the thermal vibrations are caused by the energy transfer of the illuminating electrons, this phase change would be dependent on the dose rate. Such an experiment can be done by focal series exit wave reconstruction in a sub-Angstrom electron microscope.

The observation of the dose rate dependence of the phase shift of single atoms was observed first for single Rhodium atoms [26].

Figure 4 shows the experimentally determined phase of various atoms as determined from exit 
waves reconstructed from experimental focal series of $\mathrm{Au}, \mathrm{Ge}$, and $\mathrm{MgO}$ obtained with the TEAM 0.5 HRTEM. Since the samples are thin crystalline objects observed along a zone axis, the incident electron "sees" a column of atoms. The phase shift per atom is then determined by dividing the total phase shift by the number of atoms in the column. The experimental details will be reported in [30]. When lowering the dose rate, the phase shifts are increased with respective factors 2.7, 2.1 and 1.3 which are of the same order as the "Stobbs factor". The measured atom displacements are determined by fitting and they are much larger than the usual Debye-Waller factors. For Ge and Au they are consistent with previous results [27, 31]. At very low dose the phase detection is probably limited by mechanical vibrations of the microscope (Team 0.5).As mentioned in ref [25] there is a wide variety of experiments that profit from the low dose-rate approach. They currently address catalysis in the context of artificial photosynthetic systems or tomography. Upcoming papers are listed in ref [34]. However is really important to utilize high performance microscopes to observe the effect as described in [35].

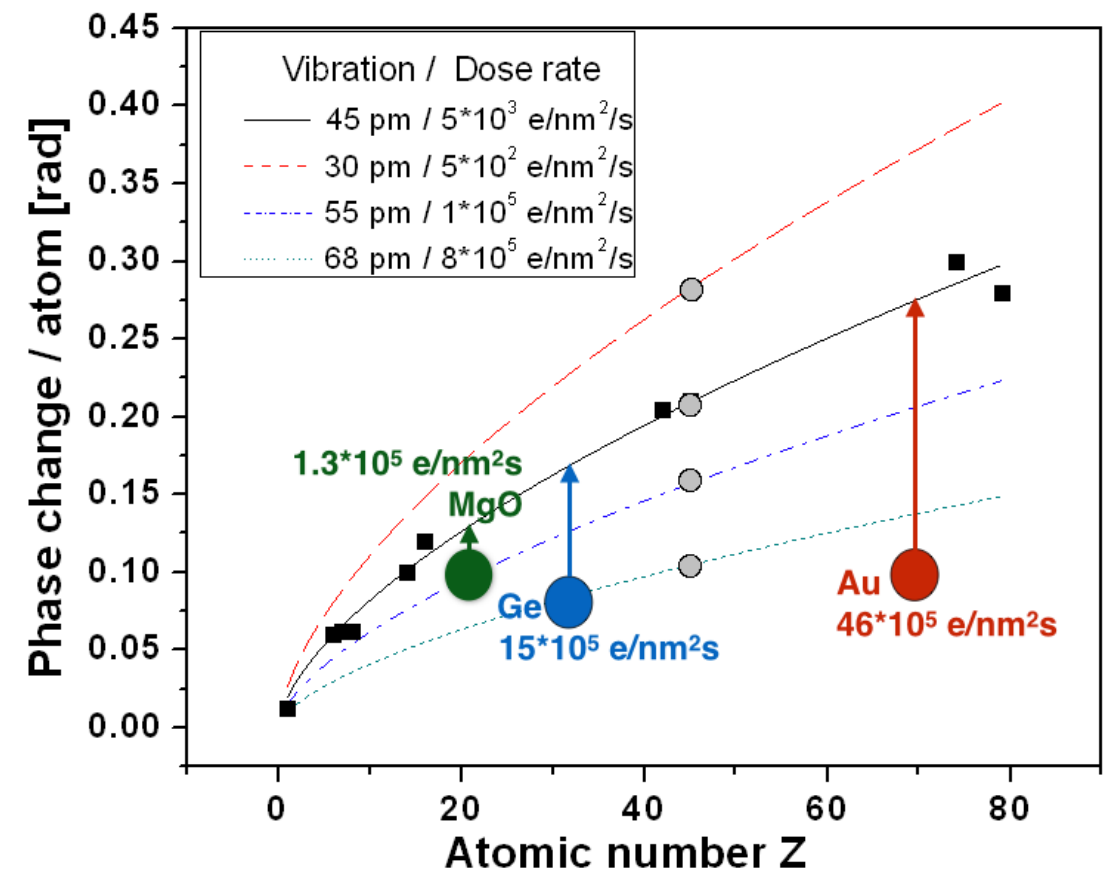

Figure 4. Experimentally observed phase shift of the electron wave when traversing a single atom for different atom types. The vibration displacement is determined by fitting with a gausssian function. When the dose rate is reduced, the phase shift increases. The solid line corresponds with the intrinsic phase detection limits of the Team 0.5 microscope probably limited by mechanical vibrations.

\section{Conclusions}

In this paper the hypothesis was put forward that, in the microscopic samples that are observed in HRTEM, the energy transferred by the illuminating electrons can be very high so as to increase the local temperature of the sample and the thermal vibration of the atoms. This increased displacement can provide an explanation for the reduction in contrast known as the "Stobbs factor". Monte Carlo simulations for the case of graphene show that at high temperatures the displacements can become very large and reversible or irreversible relaxation processes can be 
observed. An important consequence of this consideration is that radiation damage is not an unavoidable consequence of the electron dose but can be reduced by using lower dose rates. The hypothesis was also tested experimentally by observing the phase shift of the electron wave when passing through an atom. And as expected this phase shift increases with decreasing dose rate. These results demonstrate that a controllable dose rate can be used as an extra tool to study the dynamics of temperature driven physical processes in the sample.

\section{Appendix: Quantum mechanics saves HRTEM}

From Rutherford's theory, in a collision between an electron and a free atom, the electron inevitably transfers kinetic energy to the motion of the atom. But since the mass ratio is very small, the energy transfer is also very small (in the order of meV) as compared to the kinetic energy of the incident electron. But even an interaction with very small energy transfer alters the state of the object and according to Feynman's "which-way" reasoning the inelastically scattered wave does not interfere with the unscattered elastic wave. Therefore one can wonder why coherent HREM is possible anyway. The reason is that the atom is not free but bound and the energy levels for the motion of the atom are quantized. If the energy transfer stays below the lowest energy level the electron cannot transfer energy to the atom. In that case the interaction is elastic and one can make coherent images. Thus quantum mechanics saves coherent HRTEM. But beyond a certain scattering angle the interaction is unavoidably inelastic and incoherent. Thus in HREM one can only observe an atom through a "coherent window" and if one tries to push the resolution beyond this window one inevitably moves the atom. This kind of "Tantalus torment" puts a physical limit on the ultimate precision with which one can determine the position of the atom [36]. Fortunately, the electron-atom mass ratio is so small that even for the smallest energy transfer to excite a phonon (meV range) the scattering angle is large enough and the resolution of the coherent window is sufficient to resolve individual atoms.

\section{Acknowledgements:}

Ck's work was performed for the Joint Center for Artificial Photosynthesis, a DOE Energy Innovation Hub, supported through the Office of Science of the U.S. Department of Energy under Award Number DE-SC0004993. D. Van Dyck acknowledges the financial support from the Fund for Scientific Research - Flanders (FWO) under Project nos. VF04812N and G.0188.08 .

F.-R. Chen would like to thank the support from NSC 96-2628-E-007-017-MY3 and NSC 1012120-M-007-012-CC1.

\section{References}

1. D. Van Dyck. On the ultimate resolution attainable with HREM, Inst. Phys. Conf. ser. nr. 89, vol. 2, ch. 8 (1988), 349-350.

2. P.D Nellist and S.J Pennycook. The principles and interpretation of annular dark-field Zcontrast imaging, Adv. Imaging Electr. Phys. 113 (2000), 147-203; B. Forbes, A. D'Alfonso, S. Findlay, D. Van Dyck, J. LeBeau, S. Stemmer, L. Allen. Thermal diffuse 
scattering in TEM, Ultramicroscopy 111 (2011), 1670-1680.

3. M.J. Hÿtch and W.M. Stobbs. Quantitative comparison of high resolution TEM images with image simulations, Ultramicroscopy 53 (1994), 191-203.

4. C.B. Boothroyd. Why don't high-resolution simulations and images match? J. Microsc. 190 (1998), 99-108; C.B. Boothroyd. Quantification of high-resolution electron microscope images of amorphous carbon, Ultramicroscopy 83 (2000), 159-168; W. Qu, C.B. Boothroyd and A. Huan. Quantitative measurement of image intensity in transmission electron microscope images, Appl. Surf. Sci. 252 (2006), 3984-3988; C.B. Boothroyd. Quantification of energy filtered lattice images and coherent convergent beam patterns. Scanning Microsc. 11 (1997), 31-42; C.B. Boothroyd and M. Yeadon. The phonon contribution to high-resolution electron microscope images, Ultramicroscopy 96 (2003), 361-365; C.B. Boothroyd and R.E. Dunin-Borkowski. The contribution of phonon scattering to high-resolution images measured by off-axis electron holography, Ultramicroscopy 98 (2004), 115-133.

5. A. Thust. High-resolution transmission electron microscopy on an absolute contrast scale. Phys. Rev. Lett. 102 (2009), 220801.

6. D. Van Dyck. Is the frozen phonon model adequate to describe inelastic phonon scattering? Ultramicroscopy 109 (2009), 677-682.

7. Z.L. Wang. The 'frozen-lattice' approach for incoherent phonon excitation in electron scattering. How accurate is it? Acta Cryst. A54 (1998), 460-467.

8. Z.L. Wang. Thermal diffuse scattering in sub-angstrom quantitative electron microscopy phenomenon, effects and approaches, Micron 34 (3-5) (2003), 141-155.

9. J.M. Cowley. Electron microscopy of crystals with time-dependent perturbations, Acta Cryst. A44 (1988), 847-853.

10. C. Fanidis, D. Van Dyck and J. Van Landuyt. Inelastic scattering of high energy electrons in a crystal in thermal equilibrium with the environment, Ultramicroscopy 41 (1992), 5564.

11. P. Rez. Does phonon scattering give high-resolution images, Ultramicroscopy 52 (1993), 260-266.

12. A. Howie. Hunting the Stobbs factor, Ultramicroscopy 98 (2004), 73-79.

13. C.T. Koch and A. Lubk. Off-axis and inline holography: A quantitative comparison, Ultramicroscopy 110 (2010) 460-475

14. K. Du, K. von Hochmeister and F. Phillipp. Quantitative comparison of image contrast and pattern between experimental and simulated high-resolution transmission electron micrographs, Ultramicroscopy 107 (2007), 281-292.

15. J.C.H. Spence. Quantitative electron microdiffraction. J. Electr. Microsc. 45 (1996), 19-26; J.M. Zuo, J.C.H. Spence and M. O'Keefe. Bonding in GaAs, Phys. Rev. Lett. 61 (1988), 353-356.

16. J. Jansen, D. Tang, H.W. Zandbergen and H. Schenk. MSLS, a least-squares procedure for accurate crystal structure refinement from dynamical electron diffraction patterns, Acta Cryst. A54 (1998), 91-101. 
17. R. Vincent and P.A. Midgley. Double conical beam-rocking system for measurement of integrated electron diffraction intensities, Ultramicroscopy 53 (1994), 271-282.

18. R. Visser. Ph.D. Thesis, T.U. Delft (2005).

19. Z.L. Wang. Phonon scattering: how does it affect the image contrast in high-resolution transmission electron microscopy? Phil. Mag. B79 (1999), 37-48; G. Möbus, T. Gemming and P. Gumbsch. The influence of phonon scattering on HREM images, Acta Cryst. A54 (1998), 83-90.

20. J. Jinschek and C Kisielowski, private communication.

21. H. Yoshioka. Effect of inelastic waves on electron diffraction, J. Phys. Soc. Japan 12 (1957) 618-628

22. D.Van Dyck, H. Lichte and J.C.H Spence. Inelastic scattering and holography, Ultramicroscopy 81(2000), 187-194

23. D. J. Smith, A. K. Petford-Long, L. R. Wallenberg, J. O. Bovin, Dynamic AtomicLevel Rearrangements in Small Gold Particles, Science 233 (1986), 872.

24. V. G. Gayaznov, A.M. Kaprelov, A. YU. Belov, Real Temperature of Nanoparticles in Electron Microscope Beams, Phil. Mag. Lett. 63 (1991), 275-279

25. C. Kisielowski, L.-W. Wang, P. Specht, H.A. Calderon, B. Barton, B. Jiang, J.H. Kang, and R. Cieslinski. Real-time sub-Ångstrom imaging of reversible and irreversible conformations in rhodium catalysts and graphene, Phys. Rev. B 88 (2013), 024305.

26. P. Specht, R.J. Gulotty, D. Barton, R. Cieslinski, S. Rozeveld, J.H. Kang, O.D. Dubon and C. Kisielowski. Quantitative contrast evaluation of an industry-style rhodium nanocatalyst with single atom sensitivity, Chem. Cat. Chem. 3 (2011), 1034-1037.

27. D. Alloyeau, B. Freitag, S. Dag, Lin W. Wang and C. Kisielowski. Atomic resolution 3D imaging of Ge self-interstitials near a surface: Aberration-corrected transmission electron microscopy, Phys. Rev. B80 (2009), 014114.

28. C. Kisielowski, Q. Ramasse, Q.L.P. Hansen, M. Brorson, A. Carlsson, A.M. Molenbroek, H. Topsoe and S. Helveg. Imaging $\operatorname{MoS}(2)$ nanocatalysts with single-atom sensitivity, Angewandte Chemie - International edition 49 (2010), 2708-2710.

29. H. Zheng, B. Sadtler, C. Habenicht, B. Freitag, A.P. Alivisatos and C. Kisielowski. Controlling electron beam-induced structure modifications and cation exchange in cadmium sulfide-copper sulfide heterostructured nanorods, Ultramicroscopy 134 (2013), $207-213$.

30. F.-R. Chen, C. Kisielowski and D. Van Dyck, (2014), to be published.

31. C. Kisielowski and J. Jinschek. On the feasibility to investigate point defects by advanced electron microscopy, Physik Mikrostruktuierter Halbleiter 27, P. Specht, T.R. Wetherford, P. Kiesel, T. Marek, S. Malzer (eds.), Erlangen-Nuernberg (2002) 137 -144.

32. D.J. Flannigan, A.H. Zewail. 4D electron microscopy: Principles and applications, Accounts of Chemical Research 45 (2012) 1828-1839.

33. H. Zheng, J.B. Rivest, T.A. Miller, A. Timothy, B. Sadtler, A. Lindenberg, M.F. Toney, L.W. Wang, C. Kisielowski and A.P. Alivisatos. Observation of transient structural 
transformation dynamics in a $\mathrm{Cu}(2) \mathrm{S}$ nanorod, Science 333 (2011), 206-209.

34. J.A. Haber, E. Anzenburg, J. Yano, C. Kisielowski, J.M. Gregoire, Revealing the multiphase nanostructure of a quinary oxide OER electrocatalyst (2014), submitted; P. Specht, J.H. Kang, R. Cieslinski, D. Barton, B. Barton, A. Carlsson, L-W. Wang, C. Kisielowski, Direct observation of a tungsten promoted size regulation mechanism of a rhodium catalyst on alumina at atomic resolution (2014), submitted; F-R. Chen, C. Kisielowski, D. Van Dyck, Addressing Feynman's challenge - the 3D simulation of nanocrystals from single projections at atomic resolution (2014), in review.

35. C. Kisielowski, P.Specht, S. M. Gygax, B. Barton, H. A. Calderon, J. H. Kang, R. Cieslinski, Instrumental requirements for the detection of electron beam-induced object excitations at the single atom level in high-resolution transmission electron microscopy, Micron (2014), this volume.

36. D. Van Dyck. On the ultimate resolution attainable with HREM, Inst. Phys. Conf. ser. nr. 89 , vol. 2, ch. 8 (1988), 349-350. 